\title{
Infinitely many solutions for a class of sublinear fractional Schrödinger equations with indefinite potentials
}

\author{
Wen Guan ${ }^{1}$, Da-Bin Wang ${ }^{1 *}$ and Xinan Hao ${ }^{2}$
}

\section{"Correspondence:}

wangdb96@163.com

'Department of Applied

Mathematics, Lanzhou University of

Technology, Lanzhou, People's

Republic of China

Full list of author information is

available at the end of the article

\section{Springer}

\begin{abstract}
In this paper, we consider the following sublinear fractional Schrödinger equation:

$$
(-\Delta)^{s} u+V(x) u=K(x)|u|^{p-1} u, \quad x \in \mathbb{R}^{N},
$$

where $s, p \in(0,1), N>2 s,(-\Delta)^{s}$ is a fractional Laplacian operator, and $K, V$ both change sign in $\mathbb{R}^{N}$. We prove that the problem has infinitely many solutions under appropriate assumptions on $K, V$. The tool used in this paper is the symmetric mountain pass theorem.
\end{abstract}

MSC: $35 J 20 ; 35 J 60 ; 47 J 30$

Keywords: Fractional Schrödinger equation; Indefinite potential; Symmetric mountain pass theorem

\section{Introduction and main result}

In this paper, we consider the following sublinear fractional Schrödinger equation:

$$
(-\Delta)^{s} u+V(x) u=K(x)|u|^{p-1} u, \quad x \in \mathbb{R}^{N},
$$

where $s, p \in(0,1), N>2 s,(-\Delta)^{s}$ is a fractional Laplacian operator, $K, V$ both change sign in $\mathbb{R}^{N}$ and satisfy some conditions specified below.

Problem (1.1) gives the following nonlinear field equation:

$$
i \frac{\partial \Psi}{\partial t}=(-\Delta)^{s} \Psi+(1+E) \Psi-K(x)|\Psi|^{p-1} \Psi, \quad x \in \mathbb{R}^{N}, t \in \mathbb{R}^{+} .
$$

The nonlinear field Eq. (1.2) reflects the stable diffusion process of Lévy particles in random field. Later, people found that this stable diffusion of Lévy process has also a very important application in the mechanical system, flame propagation, chemical reactions in the liquid, and the anomalous diffusion of physics in the plasma. For more details, readers can refer to $[5,25,26,45]$ and the references therein.

(c) The Author(s) 2020. This article is licensed under a Creative Commons Attribution 4.0 International License, which permits use, sharing, adaptation, distribution and reproduction in any medium or format, as long as you give appropriate credit to the original author(s) and the source, provide a link to the Creative Commons licence, and indicate if changes were made. The images or other third party material in this article are included in the article's Creative Commons licence, unless indicated otherwise in a credit line to the material. If material is not included in the article's Creative Commons licence and your intended use is not permitted by statutory regulation or exceeds the permitted use, you will need to obtain permission directly from the copyright holder. To view a copy of this licence, visit http://creativecommons.org/licenses/by/4.0/. 
Problem (1.1) involves the fractional Laplacian $(-\triangle)^{s}$, which is a nonlocal operator. After this question was raised, it immediately aroused the interest of mathematicians (see $[1,4$, 6-14, 16-22, 24, 27-29, 31, 33-44, 46-55] and the references therein).

For fractional equations on the whole space $\mathbb{R}^{N}$, the main difficulty one may face is that the Sobolev embedding $H^{s}\left(\mathbb{R}^{N}\right) \hookrightarrow L^{q}\left(\mathbb{R}^{N}\right)$ is not compact for $q \in\left[2,2_{s}^{*}\right)$. To overcome this difficulty, some authors $[8,10,24,31,38,50]$ considered fractional equations with the potential $V$ satisfying the following conditions:

(V) $V \in C\left(\mathbb{R}^{N}, \mathbb{R}\right), \inf _{x \in \mathbb{R}^{N}} V(x) \geq V_{0}>0$ and, for each $M>0$, meas $\left\{x \in \mathbb{R}^{N}: V(x) \leq\right.$ $M\}<\infty$, where $V_{0}$ is a constant and meas denotes Lebesgue measure in $\mathbb{R}^{N}$.

Due to condition $(V)$, the subspace of $H^{s}\left(\mathbb{R}^{N}\right)$ embeds compactly into $L^{q}\left(\mathbb{R}^{N}\right)$ for $q \in$ $\left[2,2_{s}^{*}\right)$, which is crucial in their paper. In fact, condition $(V)$ is certain coercive condition. In the case of coercive condition $\lim _{|x| \rightarrow+\infty} V(x)=+\infty$, some authors, for example [12, 33], considered fractional equations on the whole space $\mathbb{R}^{N}$.

To overcome the difficulties caused by the lack of compactness, on the other hand, some authors restricted the energy functional to a subspace for $H^{s}\left(\mathbb{R}^{N}\right)$ of radially symmetric functions, which embeds compactly into $L^{s}\left(\mathbb{R}^{N}\right)$, for example, [9, 21, 34, 44, 54].

However, in this paper, we do not need some conditions like $(V)$ or radially symmetric. That is, our paper does not use any compact embedding on the whole space $\mathbb{R}^{N}$.

It is worth noting that, for fractional equations on the whole space $\mathbb{R}^{N}$, most results need condition $V(x) \geq 0$ (see $[1,8-10,12,13,16,18,20-22,24,28,33,34,36-38,44,50,52-54]$, in which some results were obtained in case of $V(x)=1[16,18,21,28,44])$. To the best of our knowledge, there are few results on the existence of solutions for fractional equations with a sign-changing potential except $[11,51]$. In fact, replaced $\inf _{x \in \mathbb{R}^{N}} V(x) \geq V_{0}>0$ with $\inf _{x \in \mathbb{R}^{N}} V(x)>-\infty$, condition similar to $(V)$ is needed in [11]. In [51], Xu, Wei, and Dong considered the following $p$-Laplacian equation with positive nonlinearity:

$$
(-\Delta)_{p}^{s} u+V(x)|u|^{p-2} u-\lambda|u|^{p-2} u=f(x, u)+g(x)|u|^{q-2} u, \quad x \in \mathbb{R}^{N},
$$

where $N, p \geq 2, s \in(0,1), \lambda$ is a parameter, $(-\Delta)_{p}^{s}$ is the fractional $p$-Laplacian, and $f$ : $\mathbb{R}^{N} \times \mathbb{R} \rightarrow \mathbb{R}$ is a Carathéodory function. In the case of $\lambda=0$, they obtained the existence of a nontrivial solution to this equation. Furthermore, they proved that this equation has infinitely many nontrivial solutions when $\lambda \leq 0$ or $\lambda>0$ is small enough.

In this article, we are interested in the existence of infinitely many solutions for problem (1.1) with potential function $V(x)$ changing sign in $\mathbb{R}^{N}$. Moreover, nonlinearity can be allowed to change sign. To state our main result, we assume the following:

$\left(V_{1}\right) V \in L^{\infty}\left(\mathbb{R}^{N}\right)$ and there exist $\alpha, R_{0}>0$ such that

$$
V(x) \geq \alpha, \quad \forall|x| \geq R_{0} .
$$

$\left(V_{2}\right)\left\|V^{-}\right\|_{\frac{N}{2 s}}<\frac{1}{S}$, where $V^{ \pm}(x)=\max \{ \pm V(x), 0\}$ and $S$ is the constant of Sobolev:

$$
\|u\|_{2_{s}^{*}}^{2} \leq S\|u\|_{H_{0}^{s}\left(\mathbb{R}^{N}\right)}^{2}, \quad \forall u \in H^{s}\left(\mathbb{R}^{N}\right), \text { where } 2_{s}^{*}=\frac{2 N}{N-2 s} .
$$

(K) $K \in L^{\infty}\left(\mathbb{R}^{N}\right)$ and there exist $\beta>0, R_{1}>R_{2}>0, y_{0}=\left(y_{1}, \ldots, y_{N}\right) \in \mathbb{R}^{N}$ such that

$$
K(x) \leq-\beta, \quad \forall|x|>R_{1} ; \quad K(x)>0, \quad \forall x \in B\left(y_{0}, R_{2}\right) \subset B\left(0, R_{1}\right)
$$


Our main result of this paper can be stated as follows.

Theorem 1.1 Assume $\left(V_{1}\right)-\left(V_{2}\right)$ and $(K)$ hold. Then problem (1.1) possesses infinitely many nontrivial solutions.

Remark 1.1 The ideas in this article come from the paper [3], where Schrödinger equations were considered. However, our proof is nontrivial since we present a simplified proof for the $P S$ condition by comparing to that in [3]. In fact, the PS condition was proved in [3] by concentration compactness principle. It is noticed that the PS condition plays important role in the proof of the main results in [3].

\section{Notations and preliminaries}

In this paper, we use the following notations. Let

$$
\|u\|_{q}=\left(\int_{\mathbb{R}^{N}}|u|^{q} d x\right)^{\frac{1}{q}}, \quad 1 \leq q<+\infty .
$$

Let $E$ be a Banach space and $\varphi: E \rightarrow \mathbb{R}$ be a functional of class $C^{1}$. The Fréchet derivative of $\varphi$ at $u, \varphi^{\prime}(u)$ is an element of the dual space $E^{*}$, and we denote $\varphi^{\prime}(u)$ evaluated at $v \in E$ by $\left\langle\varphi^{\prime}(u), v\right\rangle$.

Let $s \in(0,1)$, the fractional Sobolev space $H^{s}\left(\mathbb{R}^{N}\right)$ is defined by

$$
H^{s}\left(\mathbb{R}^{N}\right)=\left\{u \in L^{2}\left(\mathbb{R}^{N}\right): \frac{|u(x)-u(y)|}{|x-y|^{\frac{N}{2}+s}} \in L^{2}\left(\mathbb{R}^{N} \times \mathbb{R}^{N}\right)\right\}
$$

and endowed with the natural norm

$$
\|u\|_{H^{s}\left(\mathbb{R}^{N}\right)}=\left(\int_{\mathbb{R}^{N}}|u|^{2} d x+\int_{\mathbb{R}^{N}} \int_{\mathbb{R}^{N}} \frac{|u(x)-u(y)|^{2}}{|x-y|^{N+2 s}} d x d y\right)^{\frac{1}{2}},
$$

here

$$
[u]_{H^{s}\left(\mathbb{R}^{N}\right)}=\left(\int_{\mathbb{R}^{N}} \int_{\mathbb{R}^{N}} \frac{|u(x)-u(y)|^{2}}{|x-y|^{N+2 s}} d x d y\right)^{\frac{1}{2}}
$$

is the so-called Gagliardo (semi) norm of $u$.

Using Fourier transform, the space $H^{s}\left(\mathbb{R}^{N}\right)$ can also be defined by

$$
H^{s}\left(\mathbb{R}^{N}\right)=\left\{u \in L^{2}\left(\mathbb{R}^{N}\right): \int_{\mathbb{R}^{N}}\left(1+|\xi|^{2 s}\right)|\mathcal{F} u|^{2} d \xi<+\infty\right\}
$$

where $\mathcal{F} u$ denotes the Fourier transform of $u$.

Let $\ell$ be the Schwartz space of rapidly decreasing $C^{\infty}$ function on $\mathbb{R}^{N}, u \in \ell$, one has

$$
(-\triangle)^{s} u(x)=C(N, s) P \cdot V \cdot \int_{\mathbb{R}^{N}} \frac{u(x)-u(y)}{|x-y|^{N+2 s}} d y,
$$

the symbol $P . V$. stands for the Cauchy value, and $C(N, s)$ is a constant dependent only on the space dimension $N$ and the order $s$. 
From the results of [15], we have

$$
(-\triangle)^{s} u=\mathcal{F}^{-1}\left(|\xi|^{2 s}(\mathcal{F} u)\right) \text { for any } \xi \in \mathbb{R}^{N}
$$

Then, by Proposition 3.4 and Proposition 3.6 of [15], we have

$$
[u]_{H^{s}}^{2}=\frac{2}{C(N, s)} \int_{\mathbb{R}^{N}}|\xi|^{2 s}|\mathcal{F} u|^{2} d \xi=\frac{2}{C(N, s)}\left\|(-\Delta)^{\frac{s}{2}} u\right\|_{2}^{2} .
$$

From the above facts, the norms on $H^{s}\left(\mathbb{R}^{N}\right)$ defined as follows

$$
\begin{aligned}
u & \mapsto\left(\|u\|_{2}^{2}+\int_{\mathbb{R}^{N}}|\xi|^{2 s}|\mathcal{F} u|^{2} d \xi\right)^{\frac{1}{2}}, \\
u & \mapsto\left(\|u\|_{2}^{2}+\left\|(-\triangle)^{\frac{s}{2}} u\right\|_{2}^{2}\right)^{\frac{1}{2}}, \\
u & \mapsto u \|_{H^{s}\left(\mathbb{R}^{N}\right)}
\end{aligned}
$$

are all equivalent.

Lemma $2.1([15,30,34])$ Let $0<s<1$ such that $2 s<N$. Then there exists $C=C(n, s)$ such that

$$
\|u\|_{2_{s}^{*}} \leq C\|u\|_{H^{s}\left(\mathbb{R}^{N}\right)}
$$

for every $u \in H^{s}\left(\mathbb{R}^{N}\right)$. Moreover, the embedding $H^{s}\left(\mathbb{R}^{N}\right) \subset L^{p}\left(\mathbb{R}^{N}\right)$ is continuous for any $p \in\left[2,2_{s}^{*}\right]$ and locally compact whenever $p \in\left[2,2_{s}^{*}\right)$.

Let the homogeneous Sobolev space

$$
H_{0}^{s}\left(\mathbb{R}^{N}\right)=\left\{u \in L^{2_{s}^{*}}\left(\mathbb{R}^{N}\right):|\xi|^{s} \mathcal{F} u \in L^{2}\left(\mathbb{R}^{N}\right)\right\}
$$

This space can be equivalently defined as the completion of $C_{0}^{\infty}\left(\mathbb{R}^{N}\right)$ under the norm

$$
\|u\|_{0}^{2} \triangleq\|u\|_{H_{0}^{s}\left(\mathbb{R}^{N}\right)}^{2} \triangleq \int_{\mathbb{R}^{N}}|\xi|^{2 s}|\mathcal{F} u|^{2} d \xi
$$

The Sobolev space $E=H^{s}\left(\mathbb{R}^{N}\right) \cap L^{p+1}\left(\mathbb{R}^{N}\right)$ is endowed with the norm

$$
\|u\|=\|u\|_{0}+\|u\|_{p+1} .
$$

Obviously, $E$ is a reflexive Banach space.

The energy functional $\varphi: E \rightarrow \mathbb{R}$ corresponding to problem (1.1) is defined by

$$
\varphi(u)=\frac{1}{2} \int_{\mathbb{R}^{N}}|\xi|^{2 s}|\mathcal{F} u|^{2} d \xi+\frac{1}{2} \int_{\mathbb{R}^{N}} V(x) u^{2} d x-\frac{1}{p+1} \int_{\mathbb{R}^{N}} K(x)|u|^{p+1} d x
$$

Under our conditions, $\varphi \in C^{1}(E)$ and its critical points are solutions of problem (1.1). 
Definition 2.1 ([32]) Let $E$ be a Banach space and $A$ be a subset of $E$. Set $A$ is said to be symmetric if $u \in E$ implies $-u \in E$. For a closed symmetric set $A$ which does not contain the origin, we define a genus $\gamma(A)$ of $A$ by the smallest integer $k$ such that there exists an odd continuous mapping from $A$ to $\mathbb{R}^{k} \backslash\{0\}$. If there does not exist such $k$, we define $\gamma(A)=\infty$. We set $\gamma(\emptyset)=0$. Let $\Gamma_{k}$ denote the family of closed symmetric subsets $A$ of $E$ such that $0 \notin A$ and $\gamma(A) \geq k$.

The following result is a version of the classical symmetric mountain pass theorem [2, 32]. For the proof, please see [23].

Theorem 2.1 ([23]) Let $E$ be an infinite dimensional Banach space and $I \in C^{1}(E, \mathbb{R})$ satisfy:

$\left(I_{1}\right) I$ is even, bounded from below, $I(0)=0$, and I satisfies the Palais-Smale condition.

$\left(I_{2}\right)$ For each $k \in \mathbb{N}$, there exists $A_{k} \in \Gamma_{k}$ such that

$$
\sup _{u \in A_{k}} I(u)<0
$$

Then either of the following two conditions holds:

(i) there exists a sequence $u_{k}$ such that $I^{\prime}\left(u_{k}\right)=0, I\left(u_{k}\right)<0$ and $u_{k}$ converges to zero; or

(ii) there exist two sequences $u_{k}$ and $v_{k}$ such that $I^{\prime}\left(u_{k}\right)=0, I\left(u_{k}\right)=0, u_{k} \neq 0$, $\lim _{k \rightarrow+\infty} u_{k}=0, I^{\prime}\left(v_{k}\right)=0, I\left(v_{k}\right)<0, \lim _{k \rightarrow+\infty} I\left(v_{k}\right)=0$ and $v_{k}$ converges to a non-zero limit.

\section{Proof of Theorem 1.1}

Lemma 3.1 Suppose that $\left(V_{1}\right)-\left(V_{2}\right)$ and $(K)$ hold. Then any PS sequence of $\varphi$ is bounded in $E$.

Proof Let $\left\{u_{n}\right\} \subset E$ be such that

$$
\varphi\left(u_{n}\right) \text { is bounded and } \varphi^{\prime}\left(u_{n}\right) \rightarrow 0 \quad \text { as } n \rightarrow \infty
$$

That is, there exists $C>0$ such that $\varphi\left(u_{n}\right) \leq C$. So, according to Hölder's inequality and Sobolev's inequality, one has that

$$
\begin{aligned}
C \geq & \varphi\left(u_{n}\right)=\frac{1}{2} \int_{\mathbb{R}^{N}}|\xi|^{2 s}\left|\mathcal{F} u_{n}\right|^{2} d \xi+\frac{1}{2} \int_{\mathbb{R}^{N}} V(x) u_{n}^{2} d x-\frac{1}{p+1} \int_{\mathbb{R}^{N}} K(x)\left|u_{n}\right|^{p+1} d x \\
\geq & \frac{1}{2} \int_{\mathbb{R}^{N}}|\xi|^{2 s}\left|\mathcal{F} u_{n}\right|^{2} d \xi-\frac{1}{2} \int_{\mathbb{R}^{N}} V^{-}(x) u_{n}^{2} d x-\frac{1}{p+1} \int_{\mathbb{R}^{N}} K^{+}(x)\left|u_{n}\right|^{p+1} d x \\
\geq & \frac{1}{2}\left\|u_{n}\right\|_{0}^{2}-\frac{1}{2}\left(\int_{\mathbb{R}^{N}}\left|V^{-}\right|^{\frac{N}{2 s}} d x\right)^{\frac{2 s}{N}}\left(\int_{\mathbb{R}^{N}}\left(\left|u_{n}\right|^{2}\right)^{\frac{2_{s}^{*}}{2}} d x\right)^{\frac{2}{2_{s}^{*}}} \\
& -\frac{1}{p+1} \int_{\mathbb{R}^{N}} K^{+}(x)\left|u_{n}\right|^{p+1} d x \\
\geq & \left(\frac{1}{2}-\frac{S}{2}\left\|V^{-}\right\|_{\frac{N}{2 s}}\right)\left\|u_{n}\right\|_{0}^{2}-\frac{S^{\frac{p+1}{2}}}{p+1}\left\|K^{+}\right\|_{\frac{2_{s}^{*}}{2_{s}^{*}-(p+1)}}\left\|u_{n}\right\|_{0}^{p+1} .
\end{aligned}
$$


Since $0<p<1$, there exists $\eta>0$ such that

$$
\left\|u_{n}\right\|_{0}^{2} \leq \eta, \quad \forall n \in \mathbb{N}
$$

On the other hand, we have that

$$
\begin{aligned}
C+\frac{\left\|u_{n}\right\|}{2} \geq & \varphi\left(u_{n}\right)-\frac{1}{2}\left\langle\varphi^{\prime}\left(u_{n}\right), u_{n}\right\rangle \\
\geq & \left(\frac{1}{2}-\frac{1}{p+1}\right) \int_{\mathbb{R}^{N}} K(x)\left|u_{n}\right|^{p+1} d x \\
= & \left(\frac{1}{2}-\frac{1}{p+1}\right) \int_{\mathbb{R}^{N}} K^{+}(x)\left|u_{n}\right|^{p+1} d x+\left(\frac{1}{p+1}-\frac{1}{2}\right) \int_{\mathbb{R}^{N}} K^{-}(x)\left|u_{n}\right|^{p+1} d x \\
= & \left(\frac{1}{2}-\frac{1}{p+1}\right) \int_{\mathbb{R}^{N}}\left(K^{+}(x)+\chi_{B\left(0, R_{1}\right)}(x)\right)\left|u_{n}\right|^{p+1} d x \\
& +\left(\frac{1}{p+1}-\frac{1}{2}\right) \int_{\mathbb{R}^{N}}\left(K^{-}(x)+\chi_{B\left(0, R_{1}\right)}(x)\right)\left|u_{n}\right|^{p+1} d x,
\end{aligned}
$$

where $\|\cdot\|$ denotes the norm in $E$.

Thanks to $(K)$, we have that

$$
K^{+}(x)=0 \quad \text { for all }|x|>R_{1} \text {. }
$$

Then, by $K \in L^{\infty}\left(\mathbb{R}^{N}\right)$, we get

$$
\int_{\mathbb{R}^{N}}\left|K^{+}(x)+\chi_{B\left(0, R_{1}\right)}(x)\right|^{\frac{2_{s}^{*}}{2_{s}^{*}-(p+1)}} d x=\int_{B\left(0, R_{1}\right)}\left|K^{+}(x)+\chi_{B\left(0, R_{1}\right)}(x)\right|^{\frac{2_{s}^{*}}{2_{s}^{*}-(p+1)}} d x<\infty
$$

Hence, by Hölder's inequality and Sobolev's inequality, we have that

$$
\begin{aligned}
& \int_{\mathbb{R}^{N}}\left(K^{+}(x)+\chi_{B\left(0, R_{1}\right)}(x)\right)\left|u_{n}\right|^{p+1} d x \\
& \leq\left(\int_{\mathbb{R}^{N}}\left(K^{+}(x)+\chi_{B\left(0, R_{1}\right)}(x)\right)^{\frac{2_{s}^{*}}{2_{s}^{*}-(p+1)}} d x\right)^{\frac{2_{s}^{*}-(p+1)}{2_{s}^{*}}} \times\left(\int_{\mathbb{R}^{N}}\left(\left|u_{n}\right|^{p+1}\right)^{\frac{2_{s}^{*}}{p+1}} d x\right)^{\frac{p+1}{2_{s}^{*}}} \\
& \leq S^{\frac{p+1}{2}}\left\|K^{+}+\chi_{B\left(0, R_{1}\right)}\right\|_{\frac{2_{s}^{*}}{2_{s}^{*}-(p+1)}}\left\|u_{n}\right\|_{0}^{p+1} .
\end{aligned}
$$

Using $(K)$ again, we know that $K^{-}(x) \geq \beta$ for all $|x|>R_{1}$. Then we have that

$$
\int_{\mathbb{R}^{N}}\left(K^{-}(x)+\chi_{B\left(0, R_{1}\right)}(x)\right)\left|u_{n}\right|^{p+1} d x \geq \min (\beta, 1)\left\|u_{n}\right\|_{p+1}^{p+1}
$$

According to (3.1), (3.2), and (3.3), there exists a constant $C_{1}>0$ such that

$$
\left\|u_{n}\right\|_{p+1}^{p+1} \leq C_{1}+C_{1}\left\|u_{n}\right\|_{p+1} \quad \text { for all } n \in \mathbb{N}
$$

Since $0<p<1$, there exists a constant $C_{2}>0$ such that

$$
\left\|u_{n}\right\|_{p+1} \leq C_{2}, \quad \forall n \in \mathbb{N}
$$


Hence, it follows from (3.1) and (3.4) that $\left\{u_{n}\right\}$ is bounded in $E$.

Lemma 3.2 Suppose that $\left(V_{1}\right)-\left(V_{2}\right)$ and $(K)$ hold. Then $\varphi$ satisfies the PS condition on E.

Proof Let $\left\{u_{n}\right\} \subset E$ be such that

$$
\varphi\left(u_{n}\right) \text { is bounded and } \varphi^{\prime}\left(u_{n}\right) \rightarrow 0 \text { as } n \rightarrow \infty \text {. }
$$

By Lemma 3.1, $\left\{u_{n}\right\}$ is bounded in $E$. Going if necessary to a subsequence, from Lemma 2.1 we can assume that

$$
u_{n} \rightarrow u \text { in } E ; \quad u_{n} \rightarrow u \text { in } L_{\text {loc }}^{q}\left(\mathbb{R}^{N}\right), \quad 2 \leq q<2_{s}^{*} ; \quad u_{n} \rightarrow u \text { a.e in } \mathbb{R}^{N} .
$$

So, $\forall \psi \in C_{0}^{\infty}\left(\mathbb{R}^{N}\right)$, we have

$$
\int_{\mathbb{R}^{N}}|\xi|^{2 s} \mathcal{F} u_{n} \mathcal{F} \psi d \xi+\int_{\mathbb{R}^{N}} V(x) u_{n} \psi d x \rightarrow \int_{\mathbb{R}^{N}}|\xi|^{2 s} \mathcal{F} u \mathcal{F} \psi d \xi+\int_{\mathbb{R}^{N}} V(x) u \psi d x
$$

By $u_{n} \rightarrow u$ in $L^{p+1}(\operatorname{supp}(\psi))[15,30]$ and Lebesgue's dominated convergence theorem, one has that

$$
\int_{\mathbb{R}^{N}} K(x)\left|u_{n}\right|^{p-1} u_{n} \psi d x \rightarrow \int_{\mathbb{R}^{N}} K(x)|u|^{p-1} u \psi d x .
$$

Hence, we have

$$
0=\lim _{n \rightarrow+\infty}\left\langle\varphi^{\prime}\left(u_{n}\right), \psi\right\rangle=\left\langle\varphi^{\prime}(u), \psi\right\rangle, \quad \forall \psi \in C_{0}^{\infty}\left(\mathbb{R}^{N}\right)
$$

Then

$$
\left\langle\varphi^{\prime}(u), u\right\rangle=0
$$

Let $v_{n}=u_{n}-u$, then $u_{n}=v_{n}+u$, we have that

$$
\begin{aligned}
\left\langle\varphi^{\prime}\left(u_{n}\right), u_{n}\right\rangle= & \int_{\mathbb{R}^{N}}|\xi|^{2 s}\left|\mathcal{F} u_{n}\right|^{2} d \xi+\int_{\mathbb{R}^{N}} V(x) u_{n}^{2} d x-\int_{\mathbb{R}^{N}} K(x)\left|u_{n}\right|^{p+1} d x \\
= & \int_{\mathbb{R}^{N}}|\xi|^{2 s}\left(\left|\mathcal{F} v_{n}\right|^{2}+|\mathcal{F} u|^{2}+2 \mathcal{F} v_{n} \mathcal{F} u\right) d \xi \\
& +\int_{\mathbb{R}^{N}}\left(V(x) v_{n}^{2}+V(x) u^{2}+2 V(x) v_{n} u\right) d x \\
& -\int_{\mathbb{R}^{N}} K(x)\left|u_{n}\right|^{p+1} d x+\int_{\mathbb{R}^{N}} K(x)|u|^{p+1} d x-\int_{\mathbb{R}^{N}} K(x)|u|^{p+1} d x \\
= & \left\langle\varphi^{\prime}(u), u\right\rangle+\int_{\mathbb{R}^{N}}|\xi|^{2 s}\left|\mathcal{F} v_{n}\right|^{2} d \xi+\int_{\mathbb{R}^{N}} V(x) v_{n}^{2} d x \\
& -\int_{\mathbb{R}^{N}} K(x)\left|u_{n}\right|^{p+1} d x+\int_{\mathbb{R}^{N}} K(x)|u|^{p+1} d x+o_{n}(1) \\
\geq & \int_{\mathbb{R}^{N}}|\xi|^{2 s}\left|\mathcal{F} v_{n}\right|^{2} d \xi-\int_{\mathbb{R}^{N}} V^{-}(x) v_{n}^{2} d x \\
& -\int_{\mathbb{R}^{N}} K(x)\left(\left|u_{n}\right|^{p+1}-|u|^{p+1}\right) d x+o_{n}(1) .
\end{aligned}
$$


Thanks to (3.5) and Lemma 4.2 in [3], we have that

$$
\lim _{n \rightarrow+\infty} \int_{\mathbb{R}^{N}} K(x)\left[\left|u_{n}\right|^{p+1}-|u|^{p+1}\right] d x=\lim _{n \rightarrow+\infty} \int_{\mathbb{R}^{N}} K(x)\left|v_{n}\right|^{p+1} d x .
$$

So, we have that

$$
\begin{aligned}
\left\langle\varphi^{\prime}\left(u_{n}\right), u_{n}\right\rangle \geq & \int_{\mathbb{R}^{N}}|\xi|^{2 s}\left|\mathcal{F} v_{n}\right|^{2} d \xi-\int_{\mathbb{R}^{N}} V^{-}(x) v_{n}^{2} d x \\
& -\int_{\mathbb{R}^{N}} K(x)\left|v_{n}\right|^{p+1} d x+o_{n}(1) \\
= & \int_{\mathbb{R}^{N}}|\xi|^{2 s}\left|\mathcal{F} v_{n}\right|^{2} d \xi-\int_{\mathbb{R}^{N}} V^{-}(x) v_{n}^{2} d x \\
& -\int_{\mathbb{R}^{N}}\left(K^{+}(x)+\chi_{B\left(0, R_{1}\right)}(x)\right)\left|v_{n}\right|^{p+1} d x \\
& +\int_{\mathbb{R}^{N}}\left(K^{-}(x)+\chi_{B\left(0, R_{1}\right)}(x)\right)\left|v_{n}\right|^{p+1} d x+o_{n}(1) .
\end{aligned}
$$

Claim $1 \int_{\mathbb{R}^{N}} V^{-}(x) v_{n}^{2} d x \rightarrow 0$ as $n \rightarrow+\infty$.

In fact, by $\left(V_{1}\right)$, we have that $V^{-}(x)=0$ for all $|x| \geq R_{0}$. So, from $v_{n} \rightarrow 0$ in $L_{\text {loc }}^{q}\left(\mathbb{R}^{N}\right)$, $2 \leq q<2_{s}^{*}$, and $V \in L^{\infty}\left(\mathbb{R}^{N}\right)$, we obtain $\int_{\mathbb{R}^{N}} V^{-}(x) v_{n}^{2} d x \rightarrow 0$ as $n \rightarrow+\infty$.

Claim $2 \int_{\mathbb{R}^{N}}\left(K^{+}(x)+\chi_{B\left(0, R_{1}\right)}(x)\right)\left|v_{n}\right|^{p+1} d x \rightarrow 0$ as $n \rightarrow+\infty$.

In fact, thanks to $(K)$, we have that $K^{+}(x)=0$ for all $|x|>R_{1}$. So, by $K \in L^{\infty}\left(\mathbb{R}^{N}\right)$ and $v_{n} \rightarrow 0$ in $L_{\text {loc }}^{q}\left(\mathbb{R}^{N}\right), 2 \leq q<2_{s}^{*}$, we get

$$
\int_{\mathbb{R}^{N}}\left(K^{+}(x)+\chi_{B\left(0, R_{1}\right)}(x)\right)\left|v_{n}\right|^{p+1} d x \rightarrow 0
$$

as $n \rightarrow+\infty$.

From Claim 1, Claim 2, (3.3), and (3.6), we obtain that

$$
0=\lim _{n \rightarrow+\infty}\left(\left\|v_{n}\right\|_{0}^{2}+\min (\beta, 1)\left\|v_{n}\right\|_{p+1}^{p+1}\right)
$$

That is, $v_{n} \rightarrow 0$ in $E$. The proof is complete.

Lemma 3.3 Assume that $\left(V_{1}\right)-\left(V_{2}\right)$ and $(K)$ hold. Then, for each $k \in \mathbb{N}$, there exists $A_{k} \in \Gamma_{k}$ such that

$$
\sup _{u \in A_{k}} \varphi(u)<0
$$

Proof The proof is based on some ideas of Kajikiya [23] and is very similar to the one contained in [3]. For readers' convenience, we give the proof. Let $R_{2}$ and $y_{0}$ be fixed as in $(K)$ and denote

$$
D\left(R_{2}\right)=\left\{\left(x_{1}, \ldots, x_{n}\right) \in \mathbb{R}^{N}:\left|x_{i}-y_{i}\right|<R_{2}, 1 \leq i \leq N\right\} .
$$


Let $k \in \mathbb{N}$ be an arbitrary number and define $n=\min \left\{n \in \mathbb{N}: n^{N} \geq k\right\}$. By planes parallel to each face of $D\left(R_{2}\right)$, let $D\left(R_{2}\right)$ be equally divided into $n^{N}$ small parts $D_{i}$ with $1 \leq i \leq n^{N}$. In fact, the length $a$ of the edge $D_{i}$ is $\frac{R_{2}}{n}$. Let $F_{i} \subset D_{i}$ be new cubes such that $F_{i}$ has the same center as that of $D_{i}$. The faces of $F_{i}$ and $D_{i}$ are parallel, and the length of the edge of $F_{i}$ is $\frac{a}{2}$. Let $\phi_{i}, 1 \leq i \leq k$, satisfy: $\operatorname{supp}\left(\phi_{i}\right) \subset D_{i} ; \operatorname{supp}\left(\phi_{i}\right) \cap \operatorname{supp}\left(\phi_{j}\right)=\emptyset(i \neq j) ; \phi_{i}(x)=1$ for $x \in F_{i} ; 0 \leq \phi_{i}(x) \leq 1$, for all $x \in \mathbb{R}^{N}$. Let

$$
\begin{aligned}
& S^{k-1}=\left\{\left(t_{1}, \ldots, t_{k}\right) \in \mathbb{R}^{k}: \max _{1 \leq i \leq k}\left|t_{i}\right|=1\right\}, \\
& W_{k}=\left\{\sum_{i=1}^{k} t_{i} \phi_{i}(x):\left(t_{1}, \ldots, t_{k}\right) \in S^{k-1}\right\} \subset E .
\end{aligned}
$$

According to the fact that the mapping $\left(t_{1}, \ldots, t_{k}\right) \rightarrow \sum_{i=1}^{k} t_{i} \phi_{i}$ from $S^{k-1}$ to $W_{k}$ is odd and homeomorphic, so $\gamma\left(W_{k}\right)=\gamma\left(S^{k-1}\right)=k$. Since $W_{k}$ is compact in $E$, then $\exists \alpha_{k}>0$ such that

$$
\|u\|^{2} \leq \alpha_{k}, \quad \forall u \in W_{k} .
$$

On the other hand, by Hölder's inequality and Sobolev's embedding, we have that

$$
\|u\|_{2} \leq c\|u\|_{0}^{r}\|u\|_{p+1}^{1-r} \leq c\|u\|
$$

where $r=\frac{2_{s}^{*}(1-p)}{2\left(2_{s}^{*}-p-1\right)}$.

According to the above facts, there exists $c_{k}>0$ such that

$$
\|u\|_{2}^{2} \leq c_{k} \quad \text { for all } u \in W_{k} .
$$

Let $t>0$ and $u=\sum_{=1}^{k} t_{i} \phi_{i}(x) \in W_{k}$,

$$
\begin{aligned}
\varphi(t u) & =\frac{t^{2}}{2} \int_{\mathbb{R}^{N}}|\xi|^{2 s}|\mathcal{F} u|^{2} d \xi+\frac{t^{2}}{2} \int_{\mathbb{R}^{N}} V(x) u^{2} d x-\frac{1}{p+1} \sum_{i=1}^{k} \int_{D_{i}} K(x)\left|t t_{i} \phi_{i}\right|^{p+1} d x \\
& \leq \frac{t^{2}}{2} \alpha_{k}+\frac{t^{2}}{2}\|V\|_{\infty} c_{k}-\frac{1}{p+1} \sum_{i=1}^{k} \int_{D_{i}} K(x)\left|t t_{i} \phi_{i}\right|^{p+1} d x .
\end{aligned}
$$

From (3.7), there exists $j \in[1, k]$ such that $\left|t_{j}\right|=1$ and $\left|t_{i}\right| \leq 1$ for $i \neq j$. So

$$
\begin{aligned}
\sum_{i=1}^{k} \int_{D_{i}} K(x)\left|t t_{i} \phi_{i}\right|^{p+1} d x= & \int_{F_{j}} K(x)\left|t t_{j} \phi_{j}\right|^{p+1} d x \\
& +\int_{D_{j} \backslash F_{j}} K(x)\left|t t_{j} \phi_{j}(x)\right|^{p+1} d x+\sum_{i \neq j} \int_{D_{i}} K(x)\left|t t_{i} \phi_{i}\right|^{p+1} d x
\end{aligned}
$$

According to $\phi_{j}(x)=1$ for $x \in F_{j}$ and $\left|t_{j}\right|=1$, one has that

$$
\int_{F_{j}} K(x)\left|t t_{j} \phi_{j}\right|^{p+1} d x=|t|^{p+1} \int_{F_{j}} K(x) d x
$$


By $(K)$, one has that

$$
\int_{D_{j} \backslash F_{j}} K(x)\left|t t_{j} \phi_{j}(x)\right|^{p+1} d x+\sum_{i \neq j} \int_{D_{i}} K(x)\left|t t_{i} \phi_{i}\right|^{p+1} d x \geq 0 .
$$

According to (3.8), (3.9), (3.10), and (3.11), we have that

$$
\frac{\varphi(t u)}{t^{2}} \leq \frac{1}{2} \alpha_{k}+\frac{1}{2}\|V\|_{\infty} c_{k}-\frac{|t|^{p+1}}{(p+1) t^{2}} \inf _{1 \leq i \leq k}\left(\int_{F_{i}} K(x) d x\right)
$$

So,

$$
\lim _{t \rightarrow 0} \sup _{u \in W_{k}} \frac{\varphi(t u)}{t^{2}}=-\infty
$$

Hence, we can fix $t$ small enough such that $\sup \left\{\varphi(u), u \in A_{k}\right\}<0$, where $A_{k}=t W_{k} \in \Gamma_{k}$.

Lemma 3.4 Assume that $\left(V_{1}\right)-\left(V_{2}\right)$ and $(K)$ hold. Then $\varphi$ is bounded from below.

Proof By $(K)$, Hölder's inequality and Sobolev's embedding, as in the proof of Lemma 3.1, we have that

$$
\begin{aligned}
\varphi(u) & =\frac{1}{2}\left(\int_{\mathbb{R}^{N}}|\xi|^{2 s}|\mathcal{F} u|^{2} d \xi+\int_{\mathbb{R}^{N}} V(x) u^{2} d x\right)-\frac{1}{p+1} \int_{\mathbb{R}^{N}} K(x)|u|^{p+1} d x \\
& \geq \frac{1}{2}\left(\int_{\mathbb{R}^{N}}|\xi|^{2 s}|\mathcal{F} u|^{2} d \xi-\int_{\mathbb{R}^{N}} V^{-}(x) u^{2} d x\right)-\frac{1}{p+1} \int_{\mathbb{R}^{N}} K^{+}(x)|u|^{p+1} d x \\
& \geq\left(\frac{1}{2}-\frac{S\left\|V^{-}\right\|_{\frac{N}{2 s}}}{2}\right)\|u\|_{0}^{2}-\frac{S^{\frac{p+1}{2}}}{p+1}\left\|K^{+}\right\|_{\frac{2_{s}^{*}}{2_{s}^{*}-p-1}}\|u\|_{0}^{p+1} .
\end{aligned}
$$

Since $0<p<1$, we conclude the proof.

Proof of Theorem 1.1 In fact, $\varphi(0)=0$ and $\varphi$ is an even functional. Then by Lemmas 3.2, 3.3, and 3.4, conditions $\left(I_{1}\right)$ and $\left(I_{2}\right)$ of Theorem 2.1 are satisfied. Therefore, by Theorem 2.1, problem (1.1) possesses infinitely many nontrivial solutions converging to 0 with negative energy.

Acknowledgements

The authors are thankful to the honorable reviewers and editors for their valuable reviewing of the manuscript.

Funding

The paper is supported by the Natural Science Foundation of China (Grant nos. 11561043 and 11501318).

\section{Availability of data and materials}

Data sharing not applicable to this article as no datasets were generated or analyzed during the current study.

Competing interests

The authors declare that they have no competing interests.

Authors' contributions

All the authors have the same contribution. All authors read and approved the final manuscript.

\section{Author details}

${ }^{1}$ Department of Applied Mathematics, Lanzhou University of Technology, Lanzhou, People's Republic of China. ${ }^{2}$ School of Mathematical Sciences, Qufu Normal University, Qufu, People's Republic of China. 


\section{Publisher's Note}

Springer Nature remains neutral with regard to jurisdictional claims in published maps and institutional affiliations.

\section{Received: 3 October 2019 Accepted: 28 February 2020 Published online: 10 March 2020}

\section{References}

1. Alves, C.O., Miyagaki, O.H.: Existence and concentration of solution for a class of fractional elliptic equation in $\mathbb{R}^{N}$ via penalization method. Calc. Var. Partial Differ. Equ. 55, Article ID 47 (2016). https://doi.org/10.1007/s00526-016-0983-x

2. Ambrosetti, A., Rabinowitz, P.H.: Dual variational methods in critical point theory and applications. J. Funct. Anal. 14, 349-381 (1973)

3. Bahrouni, A., Ounaies, H., Rădulescu, V.D.: Infinitely many solutions for a class of sublinear Schrödinger equations with indefinite potential. Proc. R. Soc. Edinb., Sect. A 145, 445-465 (2015)

4. Barrios, B., Colorado, E., de Pablo, A., Sánchez, U.: On some critical problems for the fractional Laplacian operator. J. Differ. Equ. 252, 6133-6162 (2011)

5. Bertoin, J.: Lévy Processes. Cambridge Tracts in Mathematics, vol. 121. Cambridge University Press, Cambridge (1996)

6. Binlin, Z., Molica Bisci, G., Servadei, R.: Superlinear nonlocal fractional problems with infinitely many solutions. Nonlinearity 28, 2247-2264 (2015)

7. Caffarelli, L., Silvestre, L.: An extension problem related to the fractional Laplacian. Commun. Partial Differ. Equ. 32 $1245-1260$ (2007)

8. Chang, X: Ground states of some fractional Schrodinger equations on $\mathbb{R}^{N}$. Proc. Edinb. Math. Soc. 58, 305-321 (2015)

9. Chang, $X$., Wang, Z:: Ground state of scalar field equations involving a fractional Laplacian with general nonlinearity. Nonlinearity 26, 479-494 (2013)

10. Chen, C.: Infinitely many solutions for fractional Schrödinger equations in $\mathbb{R}^{N}$. Electron. J. Differ. Equ. 2016, Article ID 88 (2016)

11. Cheng, B., Tang, $X:$ : New existence of solutions for the fractional $p$-Laplacian equations with sign-changing potential and nonlinearity. Mediterr. J. Math. 13, 3373-3387 (2016)

12. Cheng, M.: Bound state for fractional Schrödinger equations with unbounded potential. J. Math. Phys. 53, Article ID $143507(2012)$

13. Dávila, J., del Pino, M., Wei, J.: Concentrating standing waves for the fractional nonlinear Schrödinger equation. J. Differ. Equ. 256, 858-892 (2014)

14. Deng, Y., Shuai, W.: Sign-changing solutions for non-local elliptic equations involving the fractional Laplacian. Adv. Differ. Equ. 1(1/2), 109-134 (2018)

15. Di Nezza, E., Palatucci, G., Valdinoci, E.: Hitchhiker's guide to the fractional Sobolev spaces. Bull. Sci. Math. 136, 521-573 (2012)

16. Dipierro, S., Palatucci, G., Valdinoci, E.: Existence and symmetry results for a Schrödinger type problem involving the fractional Laplacian. Matematiche 68, 201-216 (2013)

17. Du, X., Mao, A.: Existence and multiplicity of nontrivial solutions for a class of semilinear fractional Schrödinger equations. J. Funct. Spaces 2017, Article ID 3793872 (2017). https://doi.org/10.1155/2017/3793872

18. Felmer, P., Quaas, A., Tan, J.: Positive solutions of nonlinear Schrödinger equation with the fractional Laplacian. Proc. R. Soc. Edinb., Sect. A 142, 1237-1262 (2012)

19. Figueiredo, G.M., Siciliano, G.: A multiplicity result via Ljusternick-Schnirelmann category and Morse theory for a fractional Schrödinger equation in $\mathbb{R}^{N}$. Nonlinear Differ. Equ. Appl. 23, Article ID 12 (2016)

20. Ge, B.: Multiple solutions of nonlinear Schrödinger equation with the fractional Laplacian. Nonlinear Anal., Real World Appl. 30, 236-247 (2016)

21. Gou, T., Sun, H.: Solutions of nonlinear Schrödinger equation with fractional Laplacian without the Ambrosetti-Rabinowitz condition. Appl. Math. Comput. 257, 409-416 (2015)

22. He, X., Zou, W.: Existence and concentration result for the fractional Schrödinger equations with critical nonlinearities. Calc. Var. Partial Differ. Equ. 55, Article ID 91 (2016). https://doi.org/10.1007/s00526-016-1045-0

23. Kajikiya, R.: A critical point theorem related to the symmetric mountain pass lemma and its applications to elliptic equations. J. Funct. Anal. 225, 352-370 (2005)

24. Khoutir, S., Chen, H.B.: Existence of infinitely many high energy solutions for a fractional Schrödinger equation in $\mathbb{R}^{N}$. Appl. Math. Lett. 61, 156-162 (2016)

25. Laskin, N.: Fractional quantum mechanics and Lévy path integrals. Phys. Lett. A 268, 298-305 (2000)

26. Laskin, N.: Fractional Schrödinger equation. Phys. Rev. E 66, Article ID 056108 (2002)

27. Li, Y., Wang, D.B., Zhang, J.: Sign-changing solutions for a class of $p$-Laplacian Kirchhoff-type problem with logarithmic nonlinearity. AIMS Math. 5, 2100-2112 (2020)

28. Long, W., Peng, S.J., Yang, J.: Infinitely many positive solutions and sign-changing solutions for nonlinear fractional Schrodinger equations. Discrete Contin. Dyn. Syst. 36, 175-201 (2016)

29. Molica Bisci, G., Rădulescu, V.D.: Ground state solutions of scalar field fractional Schrödinger equations. Calc. Var. Partial Differ. Equ. 54, 2985-3008 (2015)

30. Molica Bisci, G., Rădulescu, V.D., Servadei, R.: Variational Methods for Nonlocal Fractional Problems. Encyclopedia of Mathematics and Its Applications, vol. 162. Cambridge University Press, Cambridge (2016)

31. Pucci, P., Xiang, M., Zhang, B.: Multiple solutions for nonhomogeneous Schrödinger Kirchhoff type equations involving the fractional $p$-Laplacian in $\mathbb{R}^{N}$. Calc. Var. Partial Differ. Equ. 54, 2785-2806 (2015)

32. Rabinowitz, P.H.: Minimax Theorems in Critical Point Theory with Applications to Differential Equations. CBMS. Regional Conf. Ser. in Math., vol. 65. Am. Math. Soc., Providence (1986)

33. Secchi, S.: Ground state solutions for nonlinear fractional Schrödinger equations in $\mathbb{R}^{N}$. J. Math. Phys. 54, Article ID 031501 (2013)

34. Secchi, S.: On fractional Schrödinger equations in $\mathbb{R}^{N}$ without the Ambrosetti-Rabinowitz condition. Topol. Methods Nonlinear Anal. 47, 19-41 (2016)

35. Servadei, R.: Infinitely many solutions for fractional Laplace equations with subcritical nonlinearity. Contemp. Math. 595, 317-340 (2013) 
36. Shang, X.D., Zhang, J.H.: Ground states for fractional Schrödinger equations with critical growth. Nonlinearity 27, 187-207 (2014)

37. Shang, X.D., Zhang, J.H.: Concentrating solutions of nonlinear fractional Schrödinger equation with potentials. J. Differ. Equ. 258, 1106-1128 (2015)

38. Teng, K.M.: Multiple solutions for a class of fractional Schrödinger equation in $\mathbb{R}^{N}$. Nonlinear Anal., Real World Appl. 21, 76-86 (2015)

39. Wang, D.B.: Least energy sign-changing solutions of Kirchhoff-type equation with critical growth. J. Math. Phys. 61, Article ID 011501 (2020). https://doi.org/10.1063/1.5074163

40. Wang, D.B., Li, T., Hao, X.: Least-energy sign-changing solutions for Kirchhoff-Schrödinger-Poisson systems in $\mathbb{R}^{3}$. Bound. Value Probl. 2019, Article ID 75 (2019). https://doi.org/10.1186/s13661-019-1183-3

41. Wang, D.B., Ma, Y., Guan, W.: Least energy sign-changing solutions for the fractional Schrödinger-Poisson systems in $\mathbb{R}^{3}$. Bound. Value Probl. 2019, Article ID 25 (2019). https://doi.org/10.1186/s13661-019-1128-x

42. Wang, D.B., Zhang, H., Guan, W.: Existence of least-energy sign-changing solutions for Schrödinger-Poisson system with critical growth. J. Math. Anal. Appl. 479, 2284-2301 (2019)

43. Wang, D.B., Zhang, H., Ma, Y, Guan, W.: Ground state sign-changing solutions for a class of nonlinear fractional Schrödinger-Poisson system with potential vanishing at infinity. J. Appl. Math. Comput. 61, 611-634 (2019)

44. Wang, Z., Zhou, H.S.: Radial sign-changing solution for fractional Schrödinger equation. Discrete Contin. Dyn. Syst. 36, 499-508 (2016)

45. Weitzner, H., Zaslavsky, G.M.: Some applications of fractional equations. Commun. Nonlinear Sci. Numer. Simul. 8, 273-281 (2003)

46. Wu, D.L., Li, F: Solutions for fourth-order Kirchhoff type elliptic equations involving concave-convex nonlinearities in $\mathbb{R}^{N}$. Comput. Math. Appl. 79, 489-499 (2020)

47. Wu, D.L., Yu, X.: New homoclinic orbits for Hamiltonian systems with asymptotically quadratic growth at infinity. Qual. Theory Dyn. Syst. 19, Article ID 22 (2020)

48. Xiang, M.Q., Zhang, B.L., Qiu, H.: Existence of solutions for a critical fractional Kirchhoff type problem in $\mathbb{R}^{N}$. Sci. China Math. 60, 1647-1660 (2017)

49. Xiang, M.Q., Zhang, B.L., Rădulescu, V.D.: Existence of solutions for perturbed fractional $p$-Laplacian equations. J. Differ Equ. 260, 1392-1413 (2016)

50. Xu, J., Wei, Z., Dong, W.: Existence of weak solutions for a fractional Schrödinger equation. Commun. Nonlinear Sci. Numer. Simul. 22, 1215-1222 (2015)

51. Xu, J., Wei, Z., Dong, W.: Weak solutions for a fractional $p$-Laplacian equation with sign-changing potential. Complex Var. Elliptic Equ. 61, 284-296 (2016)

52. Yang, L., Liu, Z: Multiplicity and concentration of solutions for fractional Schrödinger equation with sublinear perturbation and steep potential well. Comput. Math. Appl. 72, 1629-1640 (2016)

53. Zhang, H., Xu, J., Zhang, F: Existence and multiplicity of solutions for superlinear fractional Schrödinger equations. J. Math. Phys. 56, Article ID 091502 (2015)

54. Zhang, W., Tang, X.H., Zhang, J.: Infinitely many radial and non-radial solutions for a fractional Schrödinger equation. Comput. Math. Appl. 71, 737-747 (2016)

55. Zhang, X., Zhang, B.L., Repovš, D.: Existence and symmetry of solutions for critical fractional Schrödinger equations with bounded potentials. Nonlinear Anal. 142, 48-68 (2016)

\section{Submit your manuscript to a SpringerOpen ${ }^{\circ}$ journal and benefit from:}

- Convenient online submission

- Rigorous peer review

- Open access: articles freely available online

- High visibility within the field

- Retaining the copyright to your article

Submit your next manuscript at $\boldsymbol{~ s p r i n g e r o p e n . c o m ~}$ 